\title{
GREEN EXTRACTION OF PALMITIC ACID FROM PALM OIL USING BETAINE-BASED NATURAL DEEP EUTECTIC SOLVENTS
}

\author{
Kamarza Mulia $^{1 *}$, Dezaldi Adam ${ }^{1}$, Ida Zahrina ${ }^{2}$, Elsa Anisa Krisanti ${ }^{1}$ \\ ${ }^{1}$ Department of Chemical Engineering, Faculty of Engineering, Universitas Indonesia, Kampus UI \\ Depok, Depok 16424, Indonesia \\ ${ }^{2}$ Department of Chemical Engineering, Faculty of Engineering, University of Riau, Pekanbaru 28293, \\ Indonesia
}

(Received: December 2017 / Revised: December 2017 / Accepted: January 2018)

\begin{abstract}
In the palm oil refining processes, the free fatty acid content is reduced to an acceptable level by using steam-stripping that causes, also, the loss of nutraceuticals such as tocopherols and carotenoids. An alternative method, such as solvent extraction, to separate free fatty acids, especially palmitic acid as the major free fatty acid present in palm oil, will conserves not only the important nutraceuticals but, also, conserves energy because a steam requirement is eliminated. The objective of this research is to evaluate the performance of Natural Deep Eutectic Solvents (NADES), each consisting of betaine as the hydrogen bonding acceptor and a polyalcohol as the hydrogen bonding donor, to extract palmitic acid from palm oil. The goal is to obtain a NADES that is able to extract palmitic acid from palm oil with the highest extraction yield. The viscosity of various studied NADES was 10-236 cSt while the polarity parameter, determined by using Nile red as the solvatochromic dye, was 48.9-50.8 kcal/mol. The obtained data shows that, for NADES having similar polarity to that of betaine, the extraction yields reduce with increasing viscosity of the NADES. The highest extraction yield of $60 \%(\mathrm{w} / \mathrm{w})$, corresponding to a distribution coefficient value of 0.75 , was obtained by using NADES consisting of betaine and 1,2-butanediol. The extraction yield and distribution coefficient values indicated the potential of NADES, prepared by friendly compounds of betaine and polyalcohols, as alternative green solvents in the solvent extraction process when separating free fatty acids from palm oil.
\end{abstract}

Keywords: Betaine; Deep-eutectic-solvent; Palmitic acid; Palm-oil; Polyalcohol

\section{INTRODUCTION}

Palm oil is obtained from crude palm oil after going through the degumming, bleaching, and deacidification processes to get rid of impurities and unwanted components. Palm oil has a unique composition of saturated and unsaturated fatty acids (50:50) and contains desirable compounds such as triacylglycerols, tocopherols, tocotrienols, carotenoids and phytosterols (Edem et al., 2002). Generally, good quality oil contains more than $95 \%$ neutral triacylglycerols and $0.5 \%$ or less free fatty acids (Lin, 2002; Mba et al., 2015). The presence of free fatty acids in palm oil is undesirable because it is easily oxidized and can cause rancidity (Zahrina et al., 2018).

In some of the palm oil refining processes, valuable nutraceuticals, such as tocopherols and

\footnotetext{
*Corresponding author's email: kmulia@che.ui.ac.id, Tel: +62-21-7863516, Fax: +62-21-7863515 Permalink/DOI: https://doi.org/10.14716/ijtech.v9i2.1008
} 
carotenoids, are lost or degraded in the deacidification process because of the chemicals and high temperature steam $\left(240-260^{\circ} \mathrm{C}\right)$ used to evaporate the free fatty acids (Hamunen, 2010). It is preferable to carry out the deacidification process by using a solvent extraction method at ambient temperature and pressure because it reduces the nutraceutical compounds losses and reduces the consumption of energy (Gonçalves \& Meirelles, 2004, Rodrigues et al., 2007). However, by using hydrated ethanol as a solvent for the extraction, it turns out that there is an unacceptable low amount of extracted free fatty acids (Gonçalves et al., 2007).

Rodrigues et al. (2006) reported that free fatty acids could be extracted from cottonseed oil without reducing the nutraceutical content by using ethanol as extracting solvent. The process was optimized by adjusting the ethanol/water ratio and extraction temperature. The addition of water to ethanol solvent can reduce the nutraceutical loss present in cottonseed oil. However, this process can reduce the palmitic acid content of the palm oil to only $0.3 \%$ which is still higher than the $0.1 \%$ representing the upper limit requirement for food products. Therefore, the search for an alternative solvent for the extraction of free fatty acids from palm oil continues.

Green solvents are widely used as substitutes of hazardous organic solvents in order to minimize the environmental problems and to improve safety and health and reduce the cost $(\mathrm{Bi}$ et al., 2013). The usage of green solvents in the extraction of natural products is known as green extraction (Chemat et al., 2012). Ionic Liquids (ILs) are used in a wide range of applications (Earle \& Seddon, 2000; Earle et al., 2006; Han \& Row, 2010). However, due to the high toxicity of some ILs and the high cost of their synthesis, ILs are not commonly used in the pharmaceutical and food industries (Choi et al., 2011; Angell et al., 2012; Dai et al., 2013a).

Abbott et al. (2004) reported firstly about deep eutectic solvents that had similar physical properties and phase behaviors to of ILs. Such solvents were regarded as alternatives to ILs due to their ease of synthesis, availability, biodegradability, negligible volatility, being environmentally friendly and of low cost (Zhang et al., 2012; Hayyan et al., 2013, Maugeri et al., 2012). Deep eutectic solvents can be formed by mixing a Hydrogen Bond Acceptor (HBA), such as a quaternary ammonium salt, as and one or more Hydrogen Bond Donors (HBD) such as amides, carboxylic acids and polyalcohols. The HBA and HBD form intermolecular hydrogen bonds with each other when mixed in a certain molar ratio and produce an eutectic mixture that has a lower melting point than its individual components. Dai et al. (2013b) reported the existence of Natural Deep Eutectic Solvents (NADES) by using mixtures of various cellular constituents (primary metabolites) from all kinds of organisms. NADES, which are still liquid at room temperature, have nontoxic and environmentally friendly characteristics that are used potentially as solvents for the extraction of bioactive compounds from plants (Bi et al, 2013; Mulia et al., 2015; Garcia et al., 2016).

In this study, NADES, based on betaine as an HBA combined with various polyalcohols as HBDs, were used as a green solvent to extract free palmitic acid (representing the free fatty acids) from palm oil. The objective was to determine the composition of NADES that would be able to extract palmitic acid from palm oil with a high extraction yield and a high distribution coefficient. The ability of betaine-based NADES to extract palmitic acid was investigated by varying the type of polyalcohols as HBDs and the mole ratio of the betaine to polyalcohol. The amount of palmitic acid extracted into NADES and the physicochemical properties of the NADES, such as viscosity, polarity, and density, were determined.

\section{MATERIALS AND METHODS}

\subsection{Chemicals}

Synthesis grade palmitic acid (purity 99\%) was purchased from Merck. Palm oil (analytical grade), carotene (synthesis grade), tocopherol (synthesis grade), and glycerol (analytical grade) 
were purchased from Sigma Aldrich. Ethanol and ethylene glycol (analytical grade) were purchased from Merck. Anhydrous betaine (>98\%), synthesis grade 1,2-propanediol, 1,3propanediol, 1,2-butanediol, 1,3-butanediol, and 1,4-butanediol were purchased from Tokyo Chemical Industry (TCI). Figure 1 below shows the molecular structure of betaine, polyalcohols and palmitic acid.<smiles>CCCCCCCCCCCCCCCC(=O)O</smiles>

(3) 1,2-propanediol<smiles>CC(O)CO</smiles>

(5) 1,2-butanediol<smiles>CCC(O)CO</smiles>

(7) 1,4-butanediol

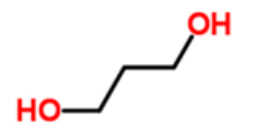

(9) Glycerol<smiles>OCC(O)CO</smiles>

(2) Betaine<smiles>C[N+](C)(C)CC(=O)[O-]</smiles>

(4) 1,3-propanediol

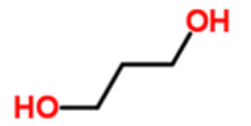

(6) 1,3-butanediol<smiles>CC(O)CCO</smiles>

(8) Ethylene Glycol<smiles>OCCO</smiles>

Figure 1 Chemical structure of palmitic acid, betaine and polyalcohols used

\subsection{Preparation of NADES and Mixture of Palm Oil}

The preparation of NADES followed the procedure reported by Dai et al. (2013b). Betaine was mixed with each of the following polyalcohols: 1,2-propanediol, 1,3-propanediol, 1,2butanediol, 1,3-butanediol, 1,4-butanediol, ethylene glycol, and glycerol; in molar ratios of 1:3, 1:4 and 1:5 respectively. A synthetic palm oil mixture was prepared according to the procedure reported by Zahrina et al. (2018); this replicates the composition of the palm oil available commercially. Mild heating was used to keep the palm oil in a liquid phase and the mixing process temperature low in order to avoid degradation of the organic materials.

\subsection{Extraction Process}

The palm oil mixture was mixed with NADES in a mass ratio of $1: 2(\mathrm{w} / \mathrm{w})$ at $50^{\circ} \mathrm{C}$ and stirred at $500 \mathrm{rpm}$ for $3 \mathrm{~h}$. The extraction was carried out in a sealed tube to prevent evaporation. The extraction temperature was kept low because, at higher temperatures, the nutriceutical compounds in palm oil, carotene and tocopherol, degrade and damage. The separation process of extracts was carried out by using centrifugation at this temperature for one hour with agitation of $3000 \mathrm{rpm}$. The temperature was kept, also, at $50^{\circ} \mathrm{C}$ during separation in order to prevent the solidification of palm oil (Israyandi et al, 2017; Zahrina et al., 2018).

\subsection{Palmitic Acid Content, Extraction Yield and Distribution Coefficient}

The amount of palmitic acid, absorbed into NADES, is considered to be equivalent to the amount of free fatty acids absorbed into NADES. The content of palmitic acid in NADES was determined based on the amount of palmitic acid that remained in the palm oil. The quantity of palmitic acid was determined by acid-base titration using potassium hydroxide. The palm oil 
was separated from NADES by washing the mixture with aquadest at $80^{\circ} \mathrm{C}$ and using a funnel separator several times. Jones' reagent was used to test the presence of NADES in the water used for washing. The palm oil, separated from NADES, was diluted in ethanol and, then, was titrated with potassium hydroxide until the phenolphtalein indicator turned light purple (Zahrina et al., 2018). The titration of the palm oil was carried in a heated flask and stirred to keep the palm oil as a liquid. As in Equation 1 below, the palmitic acid extraction yield was calculated as the percent ratio of the mass of extracted palmitic acid to the mass of palmitic acid present initially in the palm oil.

$$
\text { Palmitic acid extraction yield }(\%)=\frac{\text { mass of extracted palmitic acid }}{\text { initial mass of palmitic acid in palm oil }} \times 100
$$

The tendency of palmitic acid to transfer to the NADES phase rather than remaining in palm oil phase is expressed in Equation 2 below as a distribution coefficient $(\mathrm{K})$ in $\mathrm{mg} / \mathrm{ml}$ (NADES phase/Oil phase).

$$
\mathrm{K}=\frac{\mathrm{mg} \text { palmitic acid per } \mathrm{ml} \text { of NADES }}{\mathrm{mg} \text { palmitic acid per } \mathrm{ml} \text { of oil }}
$$

\subsection{Polarity Parameter Measurement}

The polarity of NADES, used in this study, is represented as its polar parameter calculated from the solvatochromic shift of the Nile red dye obtained by using UV-visible spectrophotometry in the 400-700 nm range (Ogihara et al., 2004). By using a Spectroquant Pharo 300 model spectrophotometer, the polarity parameter value, based on solvatochromic shift, was measured according to the procedure, reported by Dai et al. (2013a), at room temperature. As shown in Equation 3 below, the polarity parameter of a sample using Nile red dye absorbance was calculated as molar transition energy $\left(\mathrm{E}_{\mathrm{NR}}\right)$ (Reichardt, 1994):

$$
\mathrm{E}_{\mathrm{NR}}(\mathrm{kcal} / \mathrm{mol})=\frac{28,591}{\lambda \max (\mathrm{nm})}
$$

where $\lambda \max$ was the wavelength at the maximum absorbance and was measured in triplicate runs. It is recognized that the higher the polarity of a solvent, the higher its $E_{N R}$ value. The concentration of the Nile red dye and the sample in the solvent was set in the range of $10^{-4}-10^{-5}$ $M$ to reduce the interaction of visible solutes (Harris, 2008). Nile red dye is soluble in ethanol and NADES gives a pink color to the solution. The $\lambda_{\max }$ observed in the UV-spectrum of the Nile red dye shifts towards a lower wavelength in a more polar solvent (Fletcher et al., 2001), and, hence, according to Equation 3, a more polar compound is indicated by a higher E $E_{N R}$ value.

\subsection{Viscosity and Density Measurement}

The viscosity of the NADES was measured by using a Cannon-Fenske Viscometer 200 and 350 suitable for 20-100 cSt and 100-500 cSt ranges, respectively. The viscosity readings were obtained by passing samples through the cavity pipe in a water bath with a controlled temperature. The density of NADES was determined by using an Anton-Parr densitometer. The ionized water was used for calibrating the viscometer and the densitometer. Relative density is the ratio between the density of the substance at a given temperature to the density of the water at the same temperature. As the reference fluid, the density of NADES and water was measured at $40^{\circ} \mathrm{C}$. For each NADES, the viscosity and density were measured in triplicate runs. 


\section{RESULTS AND DISCUSSION}

\subsection{NADES Preparation}

In this work, NADES consisting of betaine with seven types of polyalcohols as HBDs and in three molar ratios of betaine to HBDs, were tested and the results are given in Table 1 . The stable NADES was formed when the mixture was a clear and viscous liquid and without precipitation. It was found that the preparation time required to gain a homogenous and stable mixture was different for each composition. The chemical structure of HBD and physical characteristics seem to play an important role in the formation and stability of the NADES. The formation of a stable mixture of the HBA and HBD is indicated by the depression of the freezing point relative to pure compounds (Abbott et al., 2004; Harris, 2008). The time required to form a stable mixture relates to the energy required to lower the freezing point of each compound in order to obtain a lower freezing point of the mixture., As a HBA, betaine is a solid solution at room temperature and has a melting point (freezing point) of $301{ }^{\circ} \mathrm{C}$, while the polyalcohols are liquids. Among the polyalcohols used as HBDs, 1,4-butanediol has the highest freezing point of $20.1^{\circ} \mathrm{C}$. Therefore the time required to form stable NADES, made of betaine and 1,4-butanediol, was the longest.

Table 1 Composition, formation time and physical properties of betaine based NADES

\begin{tabular}{|c|c|c|c|c|c|}
\hline $\begin{array}{l}\text { HBD (polyalcohol) in } \\
\text { Betaine-NADES }\end{array}$ & $\begin{array}{l}\text { Betaine: HBD } \\
\text { molar ratio }\end{array}$ & $\begin{array}{c}\text { Stable } \\
\text { NADES } \\
\text { formed } \\
\text { (days) }\end{array}$ & $\begin{array}{l}\text { Viscosity } \\
\quad(\mathrm{cSt})\end{array}$ & $\begin{array}{c}\text { Polarity } \\
\text { parameter } \\
\mathrm{E}_{\mathrm{NR}}(\mathrm{kcal} / \mathrm{mol})\end{array}$ & $\begin{array}{c}\text { Density } \\
(\mathrm{g} / \mathrm{ml})\end{array}$ \\
\hline \multirow{3}{*}{ 1,2-propanediol } & $1: 3$ & 7 & 44.7 & 49.57 & 1.070 \\
\hline & $1: 4$ & 1 & 47.1 & 49.59 & 1.066 \\
\hline & $1: 5$ & 1 & 24.2 & 50.60 & 1.064 \\
\hline \multirow{3}{*}{ 1,3-propanediol } & $1: 3$ & 8 & 34.8 & 48.92 & 1.075 \\
\hline & $1: 4$ & 1 & 37.8 & 50.69 & 1.072 \\
\hline & $1: 5$ & 1 & 9.7 & 48.92 & 1.065 \\
\hline \multirow{3}{*}{ 1,2-butanediol } & $1: 3$ & 17 & 25.0 & 50.43 & 1.050 \\
\hline & $1: 4$ & 1 & 37.3 & 50.29 & 1.043 \\
\hline & $1: 5$ & 1 & 32.0 & 50.34 & 1.036 \\
\hline \multirow{3}{*}{ 1,3-butanediol } & $1: 3$ & 21 & 43.9 & 49.72 & 1.051 \\
\hline & $1: 4$ & 2 & 52.4 & 49.68 & 1.044 \\
\hline & $1: 5$ & 1 & 17.0 & 49.90 & 1.035 \\
\hline \multirow{3}{*}{ 1,4-butanediol } & $1: 3$ & 24 & 51.6 & 50.51 & 1.054 \\
\hline & $1: 4$ & 8 & 23.5 & 50.56 & 1.050 \\
\hline & $1: 5$ & 5 & 41.3 & 50.78 & 1.043 \\
\hline \multirow{3}{*}{ Glycerol } & $1: 3$ & 6 & 151.0 & 49.47 & 1.205 \\
\hline & $1: 4$ & 4 & 236.0 & 49.51 & 1.224 \\
\hline & $1: 5$ & 2 & 93.7 & 49.59 & 1.229 \\
\hline \multirow{3}{*}{ Ethylene glycol } & $1: 3$ & 1 & 10.0 & 50.03 & 1.120 \\
\hline & $1: 4$ & 1 & 39.4 & 50.29 & 1.126 \\
\hline & $1: 5$ & 1 & 13.2 & 50.34 & 1.122 \\
\hline
\end{tabular}


During the preparation process, it was found that, when the mole ratio of betaine to polyalcohols was 1:3, a longer chain of polyalcohols required a longer time to form stable NADES. The time required for stable NADES of 1,2 propanediol, 1,3-propanediol, glycerol and ethylene glycol was shorter when compared to the time for all butanediols NADES. This is consistent with Dai et al.'s (2013a) studies that the spatial structure of HBDs affected the stability and characteristic of NADES. However, when the mole fraction of polyalcohols was increased, the time required to form stable NADES became similar. As occurs between cholinechloride and polyalcohols, the formation of NADES can be affected by the formation of hydrogen bonds between betaine and polyalcohols (Harris, 2018). The electronegativity difference between oxygen atoms with betaine and hydrogen atoms of hydroxyl groups in polyalcohol is preferable for the formation of hydrogen bonds (Dai et al, 2013a).

\subsection{Physical Properties of NADES}

The polarity of NADES was represented by the polar parameter $E_{N R}$, obtained from solvatochromic shift of the Nile red dye. The $\lambda_{\max }$, observed in the UV-spectrum of the Nile red dye, shifts towards a lower wavelength in a more polar solvent (Fletcher et al., 2001) and, hence, according to Equation 1, a more polar compound has a higher $E_{N R}$ value. Table 1 data indicated that the change of molar ratios of betaine to polyalcohol of 1:3 to 1:5 did not have much effect on the polarity of solvents which was represented by the values of $E_{\mathrm{NR}}$ in the range of $48.92-50.78 \mathrm{kcal} / \mathrm{mol}$.

It was reported previously that the polarity of NADES related to the hydrogen bonds that occurred between HBD and HBA, either intermolecular or intramolecular. Therefore, the formation of more hydrogen bonds caused higher polarity of the solvent. However, the result of a recent study was not consistent with the previous study, using choline chloride as HBA with 1,3-butanediol as HBD, where increased amounts of choline chloride increased the polarity of the solvent (Harris, 2008). The possible explanation for this observed data is that the polarity and molar volume of pure betaine are much higher than those of polyalcohols used as HBD. Consequently, even although the amount of polyalcohols increased in NADES, there was no significant change in the polarity.

As shown in Table 1, the viscosities of the NADES, at the molar ratio betaine to HBD from 1:3 to 1:5 were measured, and the highest viscosity was found in the 1:4 molar ratio. Increasing the amount of HBD in the solvent might increase the size of ionic species due to the formation of hydrogen bonds between HBD and HBA and, hence, might reduce either its ability to flow or increase the viscosity. However, further increases to the amount of HBD might reduce the strength of hydrogen between HBD and HBA and, therefore, the free volume of solvent might increase and the viscosity might be reduced. This explanation is in agreement with the Abbot et al.'s (2004) and Harris' (2008) statements that the presence of HBA-HBD hydrogen bonding and the free volume affected the viscosity of DESs.

Table 1 also shows that the densities of NADES, prepared from betaine with 1,2-propanediol, 1,2-butanediol, 1,3-butanediol and 1,4-butanediol, increased with the increased betaine-topolyalcohols mole ratio (from 1:5 to 1:3) in NADES. This study's observation of density was in accordance with the density reported for choline chloride based DESs. This was associated with the presence of the free volume in DESs and, hence, the increase of the salt ions-to-polyalcohols molar ratios reduced the free volume and increased the density of the DESs (Harris, 2008).

\subsection{Extraction of Palmitic Acid into NADES}

After mixing palm oil and NADES for 3 hours, palm oil and NADES were centrifuged to separate NADES from palm oil. Due to the differences in their density, NADES and oil phase were separated as lower and upper phases respectively. The NADES' color was more intense since it contained more palmitic acid than the oil phase. The palm oil-NADES emulsion took a 
longer time to separate for the butanediol-NADES that had a high viscosity value. The separation time of the glycerol-NADES was the longest due to palm oil's difficulty in diffusing within the highly viscous betaine-glycerol NADES. As in Equation 1, the palmitic acid extraction yield was calculated as the percent ratio of the mass of extracted palmitic acid to the mass of palmitic acid present initially in the palm oil. Figure 2 shows the different yields of single-stage extraction of palmitic acid by using different polyalcohol as HBD in betaine based NADES. All tested betaine-polyalcohol NADESs extracted palmitic acid from palm oil with more than $30 \%(\mathrm{w} / \mathrm{w})$. The highest yield of $60 \%(\mathrm{w} / \mathrm{w})$ was obtained by using betaine-1,2butanediol NADES at an oil-to-solvent volume ratio of $1: 2$, temperature of $40^{\circ} \mathrm{C}$ and $5.6 \%$ mass of palmitic acid in the palm oil feed. The variation of mole ratios from 1:3 to 1:5 for the betaine and 1,2-butanediol NADES did not show many differences in the extraction yields. The palmitic acid extraction yield, reported in this study, is higher than the $49 \%(\mathrm{w} / \mathrm{w})$ yield obtained previously by Zahrina et al. (2018) using the betaine monohydrate and 1,2-propanediol NADES.

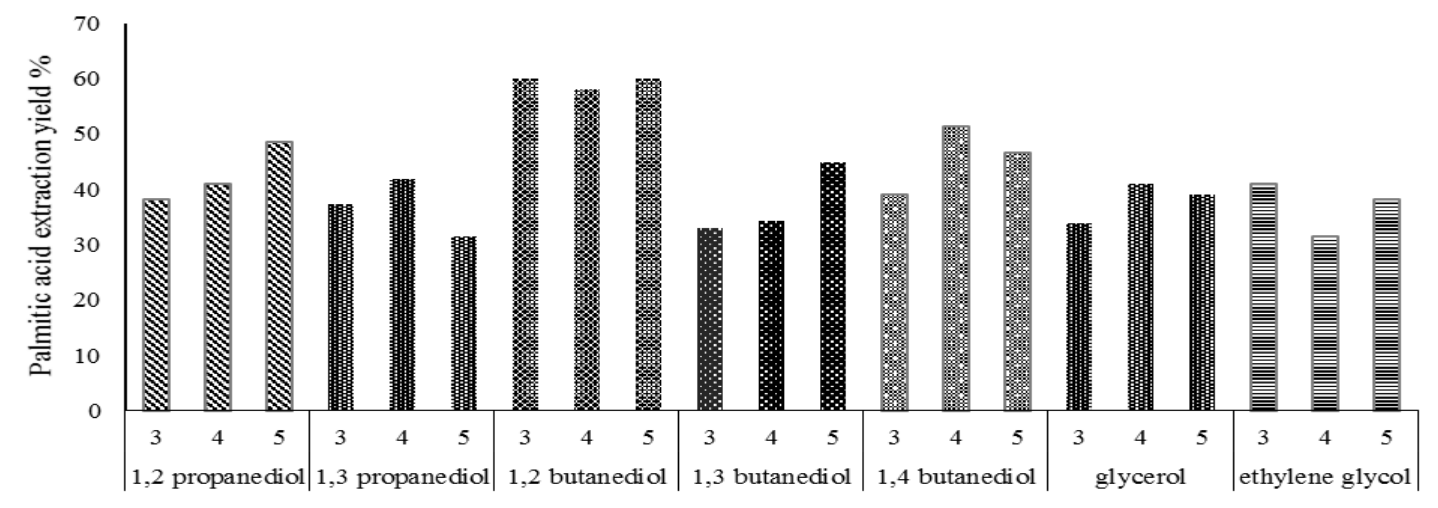

Figure 2 Palmitic acid xxtraction yield in various NADES with betaine to-HBD mole ratios $1: 3$ to $1: 5$

Although, 1,2 propanediol and 1,2-butanediol HBDs with molar ratio 1:3 have similar positions of hydroxyl groups, the viscosity of 1,2 propanediol is higher than 1,2-butanediol NADES (Table 1). This causes the extraction yield of 1,2-butanediol NADES to be higher than 1,2propanediol NADES at a similar molar ratio of 1:3. This result may be because of the larger free volume of 1,2-butanediol, with longer chain, than 1,2-propanediol in betaine NADES. Viscosity data in Table 1 and extraction yield in Figure 2 indicate that viscosity is not the only factor affecting the extraction. This is exemplified by the similar extraction yields obtained using viscous NADES with glycerol HBD, and less viscous NADES with ethylene glycol HBD. Glycerol has three hydroxyl groups and, with a shorter chain, ethylene glycol has only two hydroxyl groups. It seems that, in NADES, the spatial structure of these HBDs hinders the interaction of extracted compounds with solvent molecules. Harris (2008) reported similar results reported by using choline chloride based NADES.

Another important factor in solvent extraction is the match between the polarity of the solvent and the compound to be extracted. The measured $E_{N R}$ of palmitic acid was $51.84 \mathrm{kcal} / \mathrm{mole}$, similar to those of NADES containing 1,2-butanediol, 1,4-butanediol, and ethylene glycol as HBD (Table 1). It was noted that, when extraction yields of these NADES were plotted against their viscosities, a consistent declining trend was observed as shown in Figure 3. It appeared that the effect of viscosity was significant when the polarity parameter of the used palmitic acid and NADES were similar. The betaine-ethylene glycol NADES' lower ability to extract palmitic acid might be caused by the spatial effect. The adjacent two hydroxyl groups in ethylene glycol caused the NADES structure to become tight and rigid with limited of free 
volume. In contrast, 1,2-butanediol had an ethyl branch that enabled the NADES structure to have the unrigid conformation and let the palmitic acid diffuse and interact with the betaine. Harris (2008) reported similar situations when using NADES with choline chloride as a quaternary ammonium salt.

In the extraction process using NADES, the intermolecular formation of hydrogen bonds of the hydroxyl group of the HBD compete with the ionic oxygen of HBA and the formation of hydrogen bonds between the extracted compound compete with the ionic species of NADES. As explained in Equation 2, the tendency of palmitic acid to transfer to the NADES phase rather than remaining in the palm oil phase is expressed as a distribution coefficient of $\mathrm{K}$ in $\mathrm{mg} / \mathrm{mL}$ (NADES phase/Oil phase).

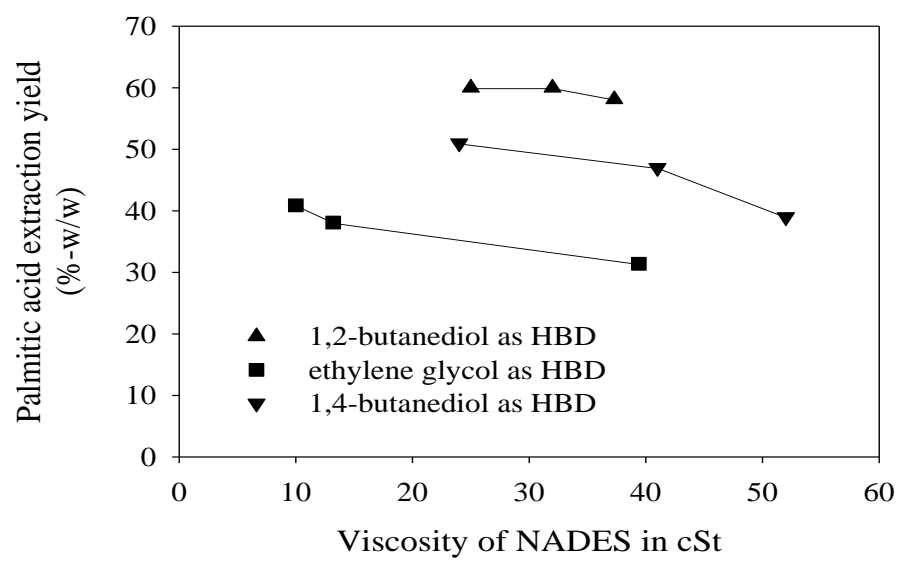

Figure 3 Palmitic acid extraction yield against viscosity of NADES with similar $E_{N R}$ values

The higher tendency to remain in the NADES phase rather than in oil phase show higher values of $\mathrm{K}$ and vice versa. Figure 4 shows that the use of different polyalcohol as HBD with betaine, in different molar ratios, resulted in a different distribution coefficient. The highest distribution coefficient of 0.75 was obtained by using 1,2-butanediol in betaine mixture. This related to $60 \%$ $(\mathrm{w} / \mathrm{w})$ yield in a single-stage extraction of palmitic acid with an oil-to-solvent volume ratio of 1:2. This result indicated that NADES, prepared by mixing 1,2-butanediol with betaine, could be used to extract palmitic oil from palm oil.

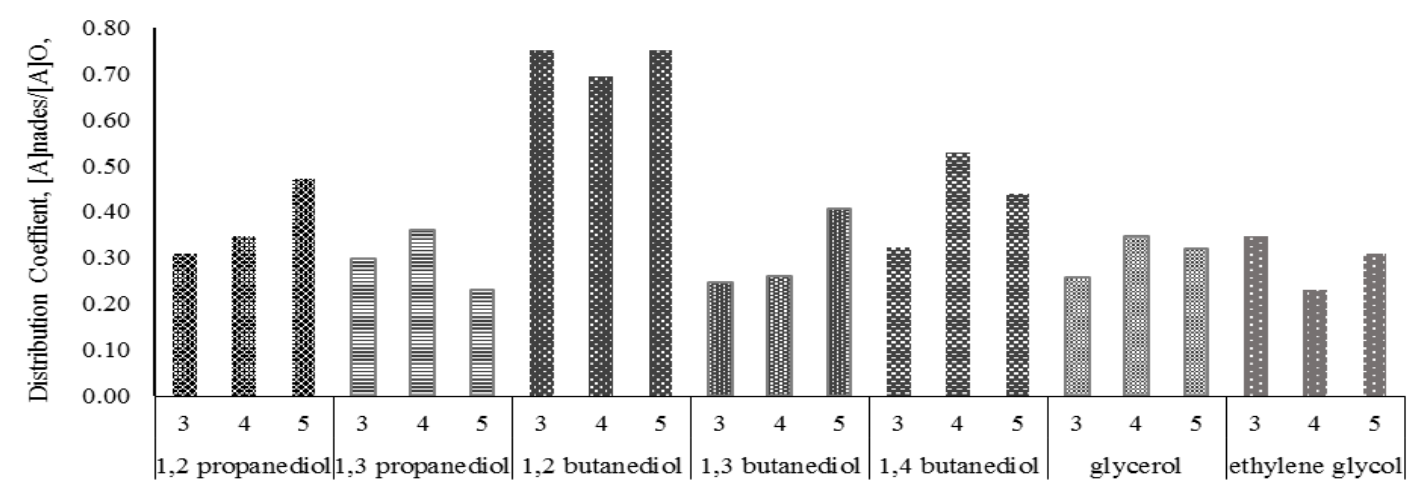

Figure 4 Distribution coefficient of palmitic acid in NADES phase/oil phase (NADES with betaine to-polyalcohol mole ratios 1:3 to $1: 5$ )

\section{CONCLUSION}

The physicochemical properties of betaine-polyalcohol NADES and the molecular structure of polyalcohols affected the stability and performance of NADES extracting palmitic acid from 
palm oil. The viscosity and density of NADES from betaine and polyalcohols appeared to be influenced by the type of polyalcohols and the molar ratios of betaine-to-polyalcohols. The hydrogen bonds and free volume formation were expected to affect the value of viscosity and density. The polarity of the studied NADES was more likely to be affected by the type of polyalcohol used rather than by the molar ratio of betaine-to-polyalcohol. There is relationship between the extraction yield and viscosity, for NADES with closed polarity $\left(\mathrm{E}_{N R}\right)$ to the polarity of pure betaine, i.e. the extraction yield declines with increasing viscosity of the NADES. The highest single-stage extraction yield of palmitic acid from palm oil was $60 \%$ (w/w) with the distribution coefficient of 0.75 ; this was obtained by using NADES from betaine and 1,2-butanediol. This study's extraction condition was mild and each NADES was prepared from environmentally friendly compounds that were less or non-toxic to human or living things. The results indicate that NADES, prepared by friendly compounds of betaine and polyalcohols, have the prospect of being alternative green solvents in separating free fatty acids from palm oils in the solvent extraction process.

\section{ACKNOWLEDGEMENT}

The authors are grateful for the Universitas Indonesia's financial support through the DRPM PITTA project, contract number 772/UN2.R3.1/HKP.05.00/2017.

\section{REFERENCES}

Abbott, A.P., Boothby, D., Capper, G., 2004. Deep Eutectic Solvents Formed between Choline Chloride and Carboxylic Acids: Versatile Alternatives to Ionic Liquids. Journal of American Chemical Society, Volume 126(29), pp. 9142-9147

Angell, C.A., Ansari, Y., Zhao, Z., 2012. Ionic Liquids: Past, Present and Future. Faraday Discussions, Volume 154, pp. 9-27

Bi, W., Tian, M., Row, K.H., 2013. Evaluation of Alcohol-based Deep Eutectic Solvent in Extraction and Determination of Flavonoids with Response Surface Methodology Optimization. Journal of Chromatography A, Volume 1285, pp. 22-30

Chemat, F., Vian, M.A., Cravotto, G., 2012. Green Extraction of Natural Products: Concept and Principles. International Journal of Molecular Sciences, Volume 13, pp. 8615-8627

Choi, Y.H., Spronsen, J.V., Dai, Y., Verberne, M., Hollmann, F., Arends, I.W.C.E, Witkamp, G.-J, Verpoorte, R., 2011. Are Natural Deep Eutectic Solvents the Missing Link in Understanding Cellular Metabolism and Physiology? Plant Physiology, Volume 156(4), pp. 1701-1705

Dai, Y., Spronsen, J., Witkamp, G.-J., Verpoorte, R., Choi, Y.H., 2013a. Natural Deep Euthectic Solvents as New Potential Media for Green Technology. Analytica Chimica Acta, Volume 766, pp. 61-68

Dai, Y., Witkamp, G.-J., Verpoorte, R., Choi, Y.H., 2013b. Natural Deep Eutectic Solvents as New Extraction Media for Phenolic Metabolites in Carthamus tinctorius L. Analytical Chemistry, Volume 85(13), pp. 6272-6278

Earle, M.J., Seddon, K.R., 2000. Ionic Liquids: Green Solvents for the Future. Pure and Applied Chemistry, Volume 72, pp. 1391-1398

Earle, M.J., Esperança, J.M., Gilea, M.A., Lopes, J.N.C., Rebelo, L.P., Magee, J.W., Seddon, K.R., Widegren, J.A., 2006. The Distillation and Volatility of Ionic Liquids. Nature, Volume 439, pp. 831-834

Edem, D., 2002. Palm Oil: Biochemical Physiological Nutritional. Plant Foods for Human Nutrition, Volume 57, pp. 319-341 
Fletcher, K.A., Storey, I.A., Hendricks, A.E., Pandey, S., 2001. Behavior of the Solvatochromic Probes Reichardt's Dye, Pyrene, Dansylamide, Nile Red and 1-pyrenecarbaldehyde within the Room Temperature Ionic Liquid PF6. Green Chemistry, Volume 3, pp. 210-215

Garcia, A., Rodriguez-Juan, E., Rodriguez-Gutierrez, G., Rios, J.J., 2016. Extraction of Phenolic Compounds from Virgin Oil by Deep Eutectic Solvents (DESs). Food Chemistry, Volume 197, pp. 554-561

Gonçalves, C.B., Meirelles, A.J.A., 2004. Liquid-liquid Equilibrium Data for the System Palm Oil + Fatty Acids + Ethanol + Water at 318.2 K. Fluid Phase Equilibria, Volume 221, pp. $139-150$

Gonçalves, C.B., Filho, P.A.P., Meirelles, A.J.A., 2007. Partition of Nutraceutical Compounds in Deacidification of Palm Oil by Solvent Extraction. Journal of Food Engineering, Volume 81, pp. 21-26

Hamunen, A., 2010. Process for Isolation of Fatty Acids, Resin Acids and Sterols from Tall Oil Pitch, US 2010/0137556 A1

Han, D., Row, K.H., 2010. Recent Applications of Ionic Liquids in Separation Technology. Molecules, Volume 15, pp. 2405-2426

Harris, R.C., 2008. Physical Properties of Alcohol Based Deep Eutectic Solvents. Ph.D. Thesis, Department of Chemistry, University of Leicester, Available online at http://hdl.handle.net/2381/4560, Accessed on 29 October 2017

Hayyan, M., Hashim, M.A, Hayyan, A., Al-Saadi, M.A, AlNashef, I.M., Mirghani, M.E.S., Saheed, O.K. 2013. Are Deep Eutectic Solvents Benign or Toxic? Chemosphere, Volume 90, pp. 2193-2195

Israyandi, Zahrina, I., Mulia, K., 2017. Optimization Process Condition for Deacidification of Palm Oil by Liquid-liquid Extraction using NADES. In: AIP Conference Proceedings 1823, 020107 (2017); doi: 10.1063/1.4978180

Lin, S.W., 2002. Palm Oil. Vegetable Oils in Food Technology. Composition Properties and Uses. In: Gunstone, F.D. (Ed), CRC Press LLC, Boca Raton, Florida. USA, pp. 59-86

Maugeri, Z., Leitner, W., de María, P.D., 2012. Practical Separation of Alcohol-ester Mixtures using Deep-Eutectic-Solvents. Tetrahedron Letters, Volume 53(51), pp. 6968-6971

Mba, O.I., Dumont, M.-J., Ngadi, M., 2015. Palm Oil: Processing. Characterization and Utilization in the Food Industry-A Review. Food Biosience, Volume 10, pp. 26-41

Mulia, K., Terahadi, F., Putri, S., Krisanti, E.A., 2015, Selected Natural Deep Eutectic Solvents for the Extraction of $\alpha$-Mangotin from Mangosteen (Garcinia mangostana L.) Pericarp. International Journal of Technology, Volume 6(70), pp. 1211-1220

Ogihara, W., Aoyama, T., Ohno, H., 2004. Polarity Measurement for Ionic Liquids Containing Dissociable Protons. Chemistry Letters, Volume 33(11), pp. 1414-1415

Reichardt, C., 1994. Solvatochromic Dyes as Solvent Polarity Indicators. Chemical Reviews, Volume 94(8), pp. 2319-2358

Rodrigues, C.E.C., Onoyama, M.M., Meirelles, A.J.A., 2006. Optimization of the Rice Bran Oil Deacidification Process by Liquid-liquid Extraction. Journal of Food Engineering, Volume 73, pp. 370-378

Rodrigues, C.E.C., Peixoto, E.C.D., Meirelles, A.J.A., 2007. Phase Equilibrium for Systems Composed by Refined Soybean Oil + Commercial Linoleic Acid + Ethanol + Water at 323.2 K. Fluid Phase Equilibria, Volume 261, pp. 122-128

Zahrina, I., Nasikin, M., Krisanti, E., Mulia, K., 2018. Deacidification of Palm Oil using Betaine Monohydrate-based Natural Deep Eutectic Solvents. Food Chemistry, Volume 240, pp. 490-495

Zhang, Q., Vigier, K.D.O., Royer, S., Je`rome, F., 2012. Deep Eutectic Solvents: Syntheses. Properties and Applications. Chemical Society Reviews, Volume 21, pp. 7108-7146 